\title{
Recommender System for Drivers of Electric Vehicles
}

\author{
João Ferreira ${ }^{1}$, Pedro Pereira ${ }^{2}$, Porfírio Filipe ${ }^{3}$ \\ GUIAA - ADEETC - ISEL \\ Lisbon, Portugal \\ $\left\{\right.$ jferreira $^{1}$, pfilipe $\left.^{3}\right\} @$ deetc.isel.ipl.pt \\ 20910@alunos.isel.ipl.pt
}

\author{
João Afonso \\ DEI - Universidade Minho \\ Guimarães, Portugal \\ jla@dei.uminho.pt
}

\begin{abstract}
Being the next big step in automobile industry, electric vehicles continue to have limited autonomy which associated with the long charging times, limited charging stations and undeveloped smart grid infrastructure demands for a hard planning of the daily use of the vehicle. This paper presents an information system that will help the driver in the daily use of his electric vehicle, minimizing the problem of range anxiety thru the continuous control of the vehicle range and presenting in time relevant information about the charging stations within reach. Given the success of recommendation systems on automatically delivering the relevant information in numerous areas of usage, it can be applied in this scenario as well as with the objective of maximizing the relevance of the information presented to the driver, which should be the strictly needed for him to make his decisions filtering out the unnecessary one.
\end{abstract}

Keywords; Recommender System; Electric Vehicle; user profile

\section{INTRODUCTION}

This paper aims to present a conceptual model for a system that meets the specific integration needs of a driver of an electric vehicle, with intelligent energy networks in order to obtain the geographic position of loading points and details about them. We propose a recommendation system that aims to present to the users the recommended loading point according to their preferences, with their usual choices and according to data collected from market allowing optimization of energy costs. The recommendation system is also used to present to the user sites and services of interest near a loading point or typical services associated with mobility in large urban areas. The system communicates with the vehicle system to obtain information on battery status. Communication with the vehicle battery allows monitoring of shipments in order to optimize costs. The integration of information recommendation system with information on the smart grid energy systems and the interface with the vehicle allows the delivery of alerts during normal vehicle use. Controlling the vehicle's range and indicating the nearest point of loading, using the geographical positioning information, using the user profile to indicate the direction of the planned route, loading preferences between fast or slow, showing energy market prices as well as identifying the proximity to public transportation scheme, are the main objectives of the system. The system provides the driver with several points of interest, (e.g. loading points, interface with public transport, parking lots with charging or tourist areas of interest), based on a recommendation system based on the profile and the preferences of the driver. The system will target a mobile device that may be a Personal Data Assistant (PDA) or a device integrated into the vehicle, taking into account the specifics of these devices have advantages such as mobility and integration with geographic positioning systems and disadvantages such as limitation of processing capacity and size of the graphic interface.

\section{SIREV CONCEPTUAL MODEL}

Being the next big step in automobile industry, electric vehicles continue to have limited autonomy which associated with the long charging times, limited charging stations and undeveloped smart grid infrastructures demands a hard planning of the daily use of the vehicle. Information systems play an important role in an information society. Given the success of recommendation systems in automatically delivering the relevant information in numerous areas of usage, it can be applied in this scenario as well as with the objective of maximizing the relevance of the information presented to the driver, which should be the strictly needed for him to make his decisions filtering out the unnecessary one. SiREV is a recommender system for $\mathrm{EV}$, with the mission of bringing the 'right' information to a mobile device. Applied to the reality of smart grids, open energy market, smart cities with increasing mobility and sustainability needs the proposed system integrates a diversity of functionalities. The main system modules are described on Figure 1: (1) GPS module: The SIREV receives the information of the geographical positioning information on the current position of the vehicle and the features that enable the calculation of distances between two points; (2) Public transportation Information module: a system with public transportation routes, schedules. The reception of such information will be made by consulting a file in XML format that contains information prepared and compiled by different operators. The file includes the geographical location of sites where you can embark on public transport scheme. The public transport information will be incorporated into items of candidate recommendation system by allocating an item property that indicates whether or not is close to public transport. This information is another dimension that enters the calculation of the usefulness of each item positively or negatively affects your score accordingly to the choices that have been made before by the driver; (3) Energy market information module: The SIREV receives the energy market discounted value of the price of energy, consulting it regularly. The only information received is the fare in the 
current format euro / $\mathrm{kWh}$ or euro value per thousand watt hours consumed. The energy market information is used to control the battery system during periods of loading. If the price of energy rises above a configurable threshold, the SIREV sends a command to the system battery to stop charging. If the price falls back to the maximum level will be sent an alert to start charging; (4) Vehicle to Grid (V2G) System Module: System that controls and monitors the status of the vehicle batteries. Collects information about the battery status at regular intervals in order to provide updated information and control the vehicle's range. The system can control battery charging in your interface providing operations to issue orders to start, pause and stop charging.

Smart Grid Module: SIREV system integrates with the smart grid in order to obtain information about the points of loading, loading types, conditions and services provided. When the charging system is directly connected to a smart grid operator and may, among other services, synchronize data of payments current account management; and (5) Charging station reserve module: One of the possible integrations between Vehicle and Energy Network (V2G) is the facility after selection, on-board system, a loading point from among the range of available battery life, communicate with the operator of that point in order to validate the availability of slots for loading and make a reservation, indicating the estimated time of arrival. In order to deal with the various issues that arise in the management of reserved slots, such as fault logging show, you must have a Reserves Management System to centralize applications, serving as a broker between operators of the loading points and LV systems.

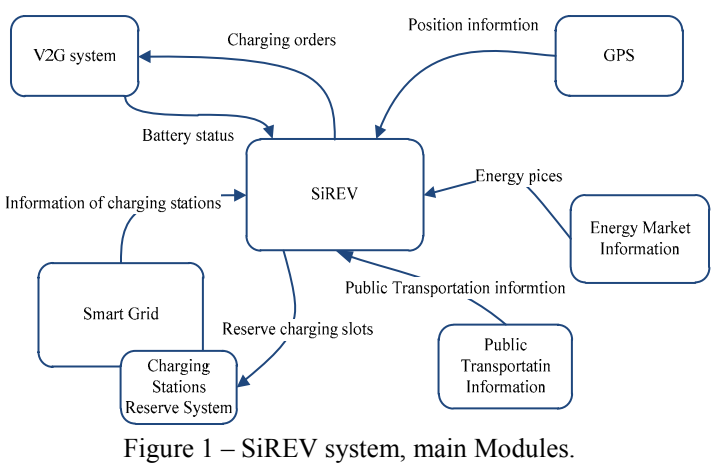

\section{RECOMMENDER ENGINE OF SIREV SYSTEM}

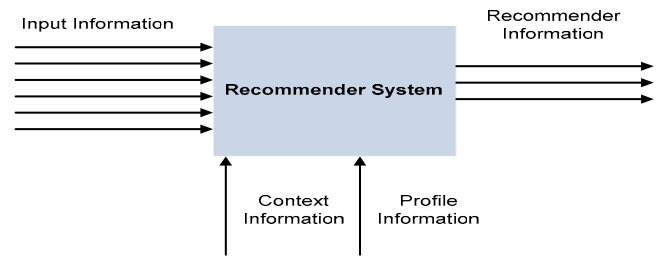

Figure 2 - Recommender Engine.

Based on the available information the SiREV system will choose (recommend) the 'right' information based on user pre-defined preferences expressed in a profile. The system uses $\mathrm{n}$ dimensions to calculate utility function (information relevance measurement) for all input information: user D1, information D2 and context D3. A diversity of recommendations is displayed. So recommended function has a new dimension $d$ (means context) and is a three dimensional vector, $u(c, d, s)$, user $\mathrm{x}$ item $\mathrm{x}$ context.

Input Information: Is the available information and represents charging places and other related information of EV driver. SiREV information modules pick and store all related information.

Profile Information - Historical data: Represents past user options in a three dimensional matrix based on selected information. In our implementation this field has only two values, one represents a user choice and zero for user disagreement.

Context Information: is used to improve recommender system performance based on the fact that user preferences change based on the context. For example in a tourism recommender system, year period changes the user's preferences among others. Several authors $[1,2]$ suggest that context information is used based on a bi-dimensional space user $\mathrm{x}$ information in a multidimensional matrix:

$u: D_{1} \times \ldots \times D_{n} \rightarrow \mathfrak{R}$, each $\mathrm{D}$ dimension is represented by an information vector and the algorithm to find the best tuple $\left(d_{1}, \ldots, d_{n}\right)$, that maximizes the utility function [3]. Our implementation uses as context information based on four main types: (1) function mode, "leisure" or "direct"; (2) day period: "morning", "afternoon" or "night"; (3) destination: "home", "work"; (4) Trip Status: "starting" "middle" e "ending". This information is stored on SiREV system and is obtained by interaction with the driver, (1) plus (3), and (2) plus (4) by the system based on pre-defined rules. So, day period is pre-defined on system, morning is until 12am, afternoon from $12 \mathrm{am}$ to $19 \mathrm{pm}$ and night the remain hours. Trip status is based on target distance, the first $25 \% \mathrm{Km}$ are considered start and last $25 \% \mathrm{Km}$ are considered ending.

Recommender information: is the SiREV system output information, ordered by a utility function. In this prereleases phase our system takes care of the charging point.

Each information type is represented by a vector, with class values. Charging point is based on:

- Charging Mode: Slow, Fast and Drop-off;

- Operator: "Mobi.e", "Better-Place", "EDP";

- Location Type: "Street", "Small Station" and "Big Station";

- Services: "without Services", "Reservation", "Washing services" and "others";

- Public Transportation Interface: "1","0";

- Nearby Point of Interest: "1","0" - only if operator mode is on travel mode.

After distance measure is introduced we calculate utility $\mu$ based on context information. For each piece of input information $\mathrm{I}_{\mathrm{i}}=\left\{\mathrm{I} 1_{\mathrm{i}}, \mathrm{I} 2_{\mathrm{i}}, \mathrm{I}_{\mathrm{i}}, \mathrm{I} 4_{\mathrm{i}}, \mathrm{C} 1_{\mathrm{i}}, \mathrm{C} 2_{\mathrm{i}}, \mathrm{C} 3_{\mathrm{i}}\right\}$, where $\left\{\mathrm{I} 1_{\mathrm{i}}, \mathrm{I} 2_{\mathrm{i}}\right.$, $\left.\mathrm{I} 3_{i}, I 4_{i}\right\}$ is the characteristics of input information $\mathrm{I}_{\mathrm{i}}$ and 
$\left\{\mathrm{C} 1_{\mathrm{i}}, \mathrm{C} 2_{\mathrm{i}}, \mathrm{C} 3_{\mathrm{i}}\right\}$ are the context characteristics. For details about the final utility function based on the nearest neighbor algorithm with context and a distance function see [4].

\section{V2G SYSTEM AND SMART GRID MODULES}

The charging of the EVs introduces a new load into the system. For every ISO, the load has a typical daily shape formed of on-peak and off-peak periods. Both the peak loads and the load shapes depend on seasonal factors - most of the summer peaking with the air conditioning loads constituting an important part of the total demand. The causal factors of the two daily peaks at around 8 to 9 a.m. and 7 to 9 p.m. are different. The morning peak is mainly caused by industrial and commercial customers while the evening peak is associated with the behavior of residential customers, who return to their homes and turn on their various appliances. A smart charging strategy should be elaborated based on production availability, distribution network limitations and contracted power and prices. Charging system is responsible to store all electricity transactions, interact with EV battery device, control electricity transaction taking in account the limitations of distribution and the driver strategy defined in a user profile. This system should support follow services: (1) Information: Battery SOC, Voltage and Amperage, $\mathrm{Km}$ to perform, store transitions data; (2) Control: Start/Stop charging, program charging, discharging.

Analyses of transactions data can be useful information for future charging or discharging process, taking in account a smart charging strategy to combine distribution network limitation and low prices. User Profile is based on, Table 1:

TABLE I. USER Profile Properties

\begin{tabular}{cc}
\hline \multicolumn{1}{c}{ Property } & Description \\
\hline User login information & User name and password \\
Drivers information & Driver Name, birth date, gender \\
Home Address & GPS position of home address \\
Work Address & GPS position of Drivers work \\
Car Information & Model, Year, Battery type and power \\
Energy market & Minimum SOC level, price to sell and to \\
behavior & buy \\
Trip information & Work days or week-end + holidays, start \\
& time, finish time, distance (km) \\
\hline
\end{tabular}

Profile operations are: Create, Change, Eliminate, Item Selection, Input Information from Tracking System. Each $\mathrm{EV}$ has a $\log$ file on a central server, where we store all charging or discharging transactions: charging place, start date, finish time, total cost. If Internet communication is available, driver can check remote the home charging process and interact if he wants. For others charging spots, SiREV system can find location and perform a reservation. SiREV system use KML file or compress KMZ, to receive data from charging stations. In this file format the EV position is defined, but we need additional information, divided in (1) Mandatory Information: operator, charging mode, charging slots and services; and (2) Additional Information: number of places, price for extra services, type of EV charging physical interface, working time. The SiREV system receives GPS coordinates of EV position and finds the nearest charging stations, showing the distance to it and giving driver guidance. Information of public transportation: SiREV needs satellite information about public transportation location and XML will be used as information Exchange file and also to receive market energy prices in $€ / \mathrm{kWh}$.

\section{SIREV SYSTEM IMPLEMENTATION}

The SiREV system is based on three main layers:

1-SiREV Aplication: SiREV system is a central information system with two main target platforms: (1) smartphones based on Windows mobile and android; and (2) vehicle dedicated system (to be considered by each manufacturer). All development was based on open source software. We focused target platforms based on Android, Google's [5] and Windows mobile operational systems.

2-Execution Services: The SiREV main services are: (1) GPS position service; (2) data storage service; (3) internet connection service; (4) SMS service; (5) user notification service.

3-Mobile Application: These layers as a group form the Mobile Platform SiREV which the target will be the mobile platform The Android platform is the platform for Google's mobile devices business, it is now one of the major operating systems used on smartphones, and the system that grew most in the market in 2009. The platform comprises an operating system, a middleware layer and a set of key applications. The development of applications for the Android platform is based on a well-documented Java API. For development of applications Google offers Android SDK that is a set of tools and documentation, integrated into the Eclipse development environment that allows the creation and test applications. The development of applications for the Android platform is based on a component-oriented architecture. A number of types of components that make up each application databases: (1) Activitie - an activitie presents a user interface and represents a specific activity of the user. An activitie may for instance be used to display a list of options to the user and capture the interaction that the user produces; (2) Service - A service has no user interface, runs in the background for an indefinite period of time. A service can be associated with one or more activities, which offer to the user the control of the application; (3) Broadcast receiver - is a component used to receive advertisements transmitted by other components and may have different origins, such as low battery announcement, announcement of change in geographical position, announcement that a photography was taken or that the user has changed the language options of the equipment; (4) Content provider - used for data exchange with others applications. All data is stored in a SQLite database; and (5) Intents - Are asynchronous messages that trigger Activities, Broadcast receivers and Services and shows receptor component.

SiREV Application: Each block in the Fig. 3 represents a set of architectural components grouped by functionality with the interfaces for communication with each other. 


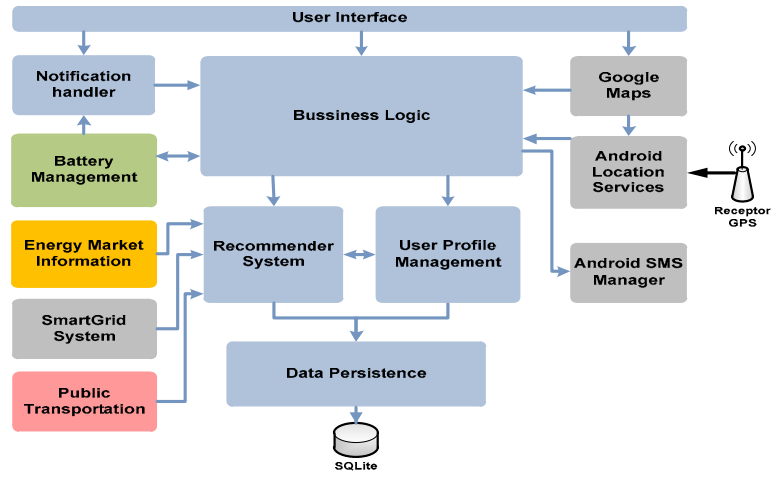

Figure 3 - SiREV system architecture

Components Model: Android architecture is based on a set of components. For implementation details see [4].

User's Interface: The android platform defines user specific interfaces called activities. In SiREV system we have one activitie for each screen functionality: (1) ProfileChooser, choose the user and associated profile; (2) ModeChooser, chooses EV usage mode (travel or working); (3) DestinationChooser home, work or some specific address; (4) MainScreen, with context data, position and SOC, see Fig. 4 (left side); and (5) RecomendationChooser, recommendation screen (Figure 4 right side);

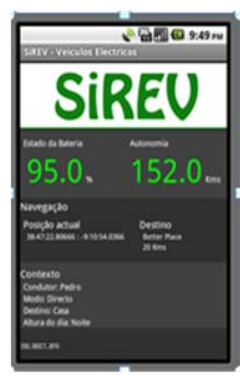

Main Application Screen

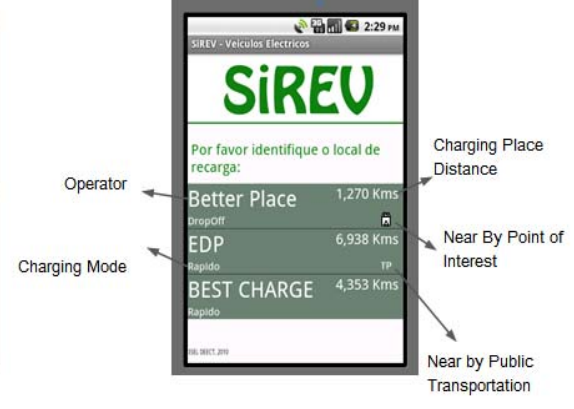

Figure 4 - Screens SiREV Application.

Business Layer: In addition to the principal components defined in the architecture there is an android type of component that is used to control application. The component is called the application and its function is to control the flow and context application user interface between different Activities that may constitute an application. The application component defines the lifetime of the application and controls the interaction of different components and subsystems that contribute to provide the functionality to the user. SIREV in this central component is called Controller, is created when the application starts and has the following definition. The controller checks application status, register the information in context and makes the connection between the presentation layer and the other services and components.

The notifications handler of sent by other subsystems or components external to the SIREV is the responsibility of the component NotificationManager.
User Profile Management: is performed by component UserProfileManager. The UserProfileManager is a Java class that aims to provide the functionality of user profile, both of registration data as historical data to be used for processing of recommendations.

Recommender System: Returns a list of Item objects and receives two lists, one with items from the historic decisions of the user and other items with the candidate. The Context object represents the current context of the system (mode of operation), the destination and time of day. Each Context object properties will be added to the list of properties of each candidate item, as explained in the definition of the algorithm. The first step in implementing the method is to create a container for the processed items that is a list sorted by the attribute value which is calculated for each item. Below is a list of items iterated candidates in order to calculate the value of each element. The calculation of utility is made by research on the history list by the item that most resembles the current item or the item that has been preferred by the user that has more characteristics in common with the item that will be the candidate for what will be shortest distance. Found this item is assigned to use the item candidate that is the inverse of distance, so that an item closer to another has chosen a more useful. Next, the item is placed in an orderly manner in the container of processed items. After completing the iteration through all the elements in the list of candidate items is created to return a list with size 3. To this list are added the first three elements of the container of processed items, which correspond to the items of most use. The method returns the list and ends.

Integration layer: The system interacts with different and heterogeneous external systems that implement different integration patterns supported on various communication protocols. For each external system identifies the most widely used standard, standard, or in case there is no set standard or universally accepted, an adapter that abstracts of one possible implementation of a standard pattern. The interface with external systems offers a complete abstraction of its implementation, with the purpose to isolate and decouple the systems. Thus our system is flexible to integrate into any type of system since the interface is observed. The integration components are components Android-type Content Providers, and implement the standard architecture Adapter [6].

Smart Grid: Information on the loading points is received in XML files in KML format which are obtained through the periodic synchronization with the smart grid. The synchronization is done via an Internet connection by going to an address configured on the system and using the classes in the API package android.net android. The component SmartGridService is a service to be launched android who continues to run in the background, performing regular updating of the information file of the loading points. The service uses the component that is a component SmartGridAdapter android type of content provider. This component has the task to interface with external system that provides data on the loading points and make the necessary 
changes to data in order to provide records that the service recognizes SmartGridService.

The battery management system consists of the following activities [4, 5]: (1) BatteryDetails: is a screen with information about the current state of the battery and the mode of operation, ie whether it is charging or running. By putting this in a different package activitie allow the screen to be customized for different constructors or battery management systems, providing data specific to each; (2) BatteryService: Android service responsible for managing and monitoring the battery. It also has the function of periodically monitor the battery status and trigger messages such as autonomy IntentBroadcast low of $50 \mathrm{Kms}$; (3) BatteryAdapter: Component responsible for integration with the system battery. As explained above implements the standard adapter that can be developed by a component of this type for any battery system of any manufacturer that has been duly specified. This component also has to deal with multiple physical interfaces to connect to the battery system. The SIREV is designed to be implemented in both systems integrated into the vehicle as smartphones. At that uses the API BateryAdapter android to become fully the physical connection to another system. It is possible to connect via USB or Ethernet in the case of an integrated mechanism in the vehicle or by Bluetooth or Wi-Fi if the SIREV run a smartphone without physical connection to the vehicle. The platform provides all the necessary mechanisms to establish the physical connection, the application just need to know the URI that will communicate.

Public Transport Information: Information on public transport is available to the system in the form of an XML file that contains position information of the geographic locations of stopping the transport. The recommendation system before processing the applicant submits the items collection of items for the PublicTransportService that identifies each item to its proximity to an interface with public transport. If so, the property nearPublicTransport item is placed with the value. The connection to an information system will be made by public transport component PublicTransportAdapter.

Energy Market Information: The energy market information will be managed by PriceService service that will periodically contact the information system of the energy market in order to update the current price. The connection to the external system is the responsibility of the adapter EnergyMarketAdapter this system that will only return a numeric value corresponding to the price of energy.

Android Services are: Position system, Mapview, SMS Manager.

\section{CONCLUSIONS}

The main objective of this work is to help drivers in daily use of EV, acting proactively and increase the attractiveness of EV. However, technology is still developing and so are all the information systems necessary for their full integration. In this work, we have proposed the integration of information from various areas that will no doubt participate in the successful implementation of a mobility model for drivers of electric vehicles. However, it was extremely difficult to find projects and technologies to mature enough for an actual operating environment. There are some projects, prototypes of the various players are mostly from universities or government projects. There are also some projects for companies weighing on the market, but which are in the process of prototyping and market testing. This difficulty to find specifications and standards for use globally accepted for exchange of information, particularly with the smart grid systems, public transport system, control systems and batteries with the electricity market, means that although functionally it is easy to explain the need and requirements of these systems, from a technical standpoint you have chosen to submit asynchronous communication modes based on open specifications and well-known case of XML. It was also necessary to ensure that the integration model is sufficiently generic and flexible to be adapted to different systems, having a low impact on the implementation of SIREV. On the other hand, was easy to see that the technologies needed to develop a mobile system capable of receiving position information and geographic connection to external devices or to the Internet, are a very advanced level of development. The Android system allows a very simple and efficient development of applications and with the advantage of being totally free of cost, both with regard to the licensing of their own API and developer tools, all based on free software. The ease of development, integration with GPS, USB, Bluetooth, SMS, and test execution makes work very efficient implementation. The usage of SIREV showed only part of all its potential for use in this context and will be part of future work to deepen the study of a more complex model of recommendation and optimized for the scenario in question. Demonstrating the validity and enforceability of this project in a real use and its added value in an attempt to identify functional requirements and identify possible ways for its implementation.

\section{REFERENCES}

[1] A. Gediminas, Toward the Next Generation of Recommender Systems: A Survey of the State-of-the-Art and Possible Extensions, IEEE Transactions on Knowledge and Data Eng, V17, N 6, June 2005

[2] J. Clerk Maxwell, A Treatise on Electricity and Magnetism, 3rd ed., vol. 2. Oxford: Clarendon, 1892, pp.68-73.

[3] A. Gediminas and A. Tuzhilin, Multidimensional Recommender Systems: A Data Warehousing Approach, Springer Berlin 2001

[4] P. Pereira, Sistema de Recomendação para Condutores de Veículos Eléctricos, Master Thesis in Informatics at ISEL, 2010. Available at www.deetc.isel.ipl.pt/Matematica/JF/sirev.pdf

[5] Android Dev Guide, http://developer.android.com/guide/index.html

[6] GangOfFour, Design Patterns: Elements of Reusable Object-Oriented Software, AddisonWesley, 1994 